\title{
Nahrungsmittelallergie à la carte
}

E $s$ ist mir eine große Freude, Ihnen in Form dieses Themenheftes von Allergo Journal einen Teil der Arbeit der Arbeitsgruppe Nahrungsmittelallergie der DGAKI vorzustellen. Wir haben für Sie Beiträge zu interessanten Fragestellungen zusammengestellt und hoffen, Sie somit zum Thema "Nahrungsmittel und Allergien" auf den aktuellsten Wissensstand zu bringen.

Der Artikel „Nahrungsmittel-Anaphylaxie: Daten aus dem AnaphylaxieRegister" präsentiert Ihnen aktuelle Zahlen des im deutschsprachigen Raum seit nunmehr fünf Jahren aktiven AnaphylaxieRegisters (S. 234). Nur durch die Unterstützung zahlreicher Allergologen aus Deutschland, Österreich und der Schweiz ist es möglich, Daten auch zur Nahrungsmittel-Anaphylaxie systematisch zu erfassen. Neben bekannten Erkenntnissen (Nahrungsmittel sind die häufigsten Auslöser schwerer allergischer Reaktionen bei Kindern) konnten wir ein breites Spektrum von Nahrungsmitteln als Auslöser einer Anaphylaxie identifizieren.

Auch Sojaprodukte, so zeigen die Daten des Anaphylaxie-Registers, sind ein häufiger Allergieauslöser. Somit bietet der Artikel aus der Arbeitsgruppe von Regina Treudler mit den vor-

„Wir haben für Sie aktuelle Beiträge zusammengestellt und hoffen, Sie damit beim Thema ,Nahrungsmittel und Allergien“ auf den aktuellen Stand zu bringen." liegenden Ausführungen eine ideale Möglichkeit, sich über die aktuellen Entwicklungen beim Thema Sojaallergie zu informieren (S. 243). Gerade Birkenpollenallergiker sind eine Risikogruppe. Dabei muss berücksichtigt werden, dass Soja in zahlreichen Produkten vorkommt, jedoch nur in eiweißreichen Produkten, wie beispielsweise Sojadrinks, häufiger ein Risiko darstellt.

Die Sojaallergie ist auch ein sehr gutes Beispiel dafür, wohin die moderne, differenzierte molekulare Allergiediagnostik führt. So kann etwa die Bestimmung von IgE gegenüber Gly $m 4$ bei einem Patienten mit einer schweren Reaktion nach

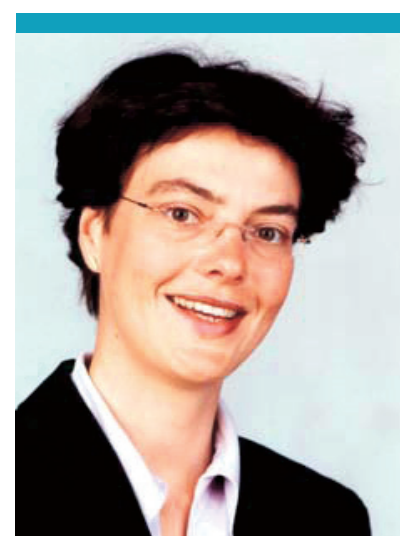

Prof. Dr. Margitta Worm, Allergie-Centrum-Charité, Klinik für Dermatologie, Venerologie und Allergologie, Charité Universitätsmedizin Berlin
Konsum eines gering prozessierten Sojaprodukts differenzialdiagnostisch weiterhelfen. Anhand von Fallbeispielen stellt Jörg Kleine-Tebbe ab Seite 251 sehr anschaulich dar, wie ein konkreter Nutzen durch die Bestimmung von Einzelallergenen in der Praxis entstehen kann.

Abgerundet wird das Themenheft durch einen Beitrag von den Kollegen Erdmann und Merk mit einem klinisch eher seltenen Thema, dem hämatogenen Kontaktekzem durch Nahrungsmittel (S. 264). Der Artikel wird Sie dabei unterstützen, auch an seltene klinische Manifestationen und deren mögliche Auslöser - und hierzu gehören auch Nahrungsmittel - zu denken.

Ich bin sicher, dass Sie viel Freude beim Lesen der Artikel haben werden, und wir hoffen zugleich, das die Informationen für Ihre tägliche Praxis einen Erkenntnisgewinn bieten!

Angenehme Lektüre wünscht Ihnen
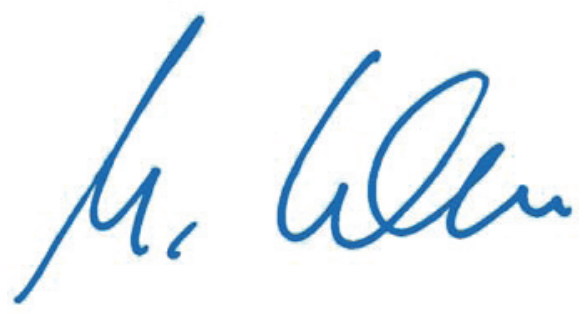

Prof. Dr. Margitta Worm für die AG Nahrungsmittelallergie der DGAKI 